\title{
Enfermedad renal en la diabetes: A propósito del día mundial del riñón
}

\author{
LEOPOLDO ARDILES $^{1}$, SERGIO MEZZANO ${ }^{1}$
}

\section{Diabetic renal disease: The World Kidney Day in Chile}

The third version of the World Kidney Day will be held on May 13, 2010 in Chile and will be focused in diabetic renal damage, the main cause of chronic kidney disease (CKD). Currently, we are living a pandemia of CKD, a progressive and irreversible condition with high social and economic impact. In Chile, we have 857 patients per million inhabitants in hemodialysis and $35 \%$ are secondary to diabetes. Our general prevalence of diabetes is $4.2 \%$, rising to $15 \%$ in people aged more than 64 years. With a $34 \%$ prevalence of hypertension, an aging population, high prevalence of obesity, and a sedentary lifestyle, there is an estimation of a rise in $85 \%$ of the prevalence of diabetes in South-America, for the next decades. The steps to be taken are clear: campaigns should be aimed at (1) prevention of type 2 diabetes; (2) screening for early diabetic kidney disease; (3) increasing patient awareness of kidney disease; (4) using medications of proven strategy and finally (5) research on new therapies. These concepts must be included in community and professional education to reduce the effects of this pandemia.

(Rev Med Chile 2010; 138: 397-400).

Key words: Diabetes complications; Kidney failure, chronic; Renal dialysis.

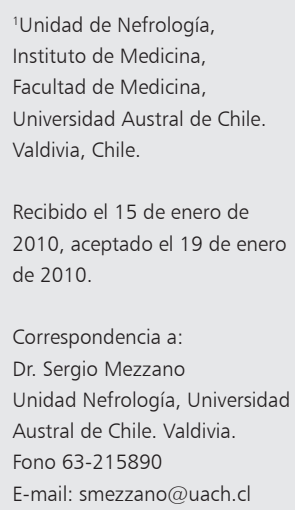

$\mathrm{E}$ 1 día mundial del riñón es una jornada que organiza anualmente la Sociedad Internacional de Nefrología y la Federación Internacional de Fundaciones del Riñón, programada para el 11 de marzo de este año y cuya tercera versión será conmemorada en Chile el 13 de mayo de 2010. En esta oportunidad la "idea fuerza" será la relación existente entre la diabetes mellitus y el daño renal, teniendo en cuenta que existe una creciente incidencia con caracteres de pandemia, en las enfermedades renales progresivas en el mundo y, en particular, en aquella derivada de la diabetes (Figura 1).

El siglo en que vivimos desafortunadamente cuenta con las condiciones ideales para el desarrollo de la diabetes ${ }^{1,2}$ y las proyecciones epidemiológicas actuales pronostican que el impacto será mayor en los países en desarrollo, esperándose para el año 2025 un incremento de $85 \%$ en el número de pacientes diabéticos en Sudamérica ${ }^{3}$.

Lamentablemente, la diabetes es una causa de enfermedad renal crónica y como tal tiene naturaleza progresiva, no es curable, se asocia a una alta morbimortalidad cardiovascular y es fuente de invalidez y costos socioeconómicos significativos para cualquier economía, especialmente las menos desarrolladas. Actualmente es la causa más frecuente de nuevos casos de enfermedad renal crónica en países desarrollados ${ }^{4}$ y lo mismo ocurre en Chile, donde representa el 35\% de los pacientes en tratamiento dialítico (XXIX cuenta de Hemodiálisis Crónica en Chile al 31 de agosto de 2009. Dr Hugo Poblete B., Sociedad Chilena de Nefrología. 2009). Estos datos son aún más preocupantes si tomamos en cuenta que la encuesta nacional de salud chilena de 2003 demostró una prevalencia general de diabetes de 4,2\% (la que asciende a $9,4 \%$ en el grupo etario de 45 a 64 años 


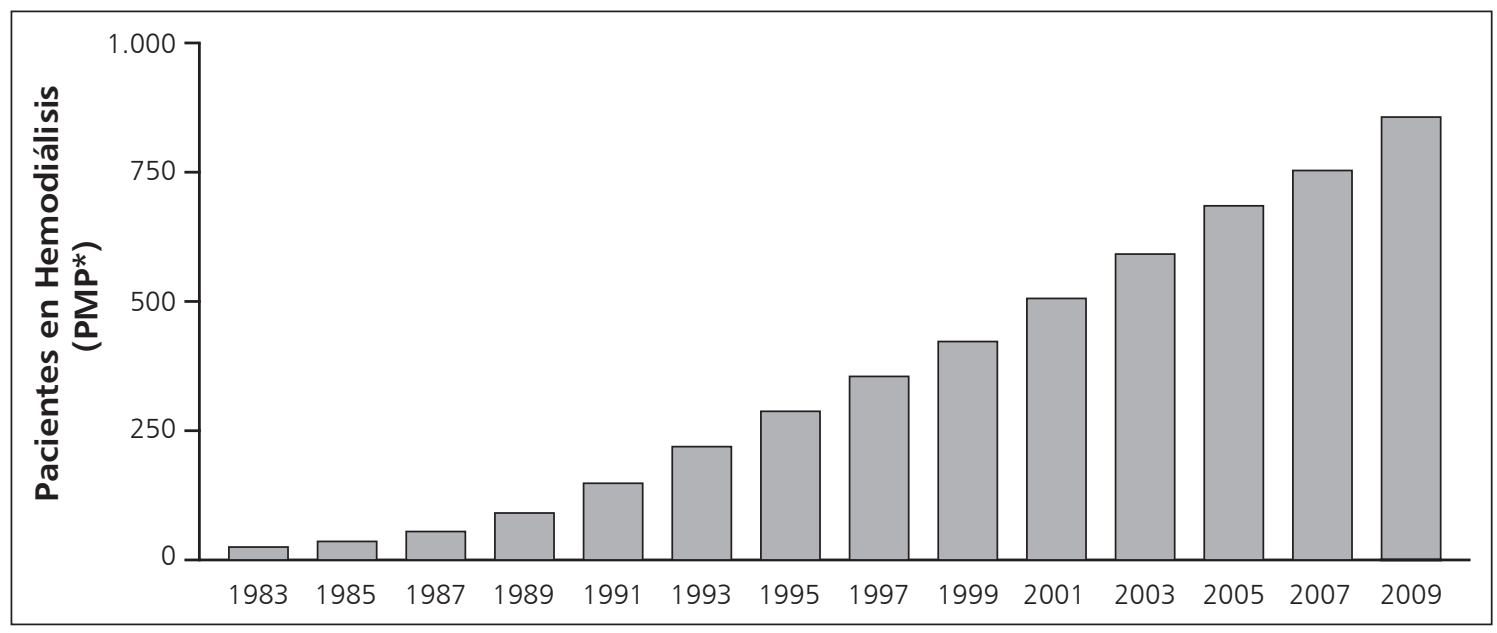

Figura 1. Incremento progresivo en la prevalencia de pacientes en hemodiálisis crónica en Chile (Año 2009: 857 PMP). Fuente: XXIX cuenta de Hemodiálisis Crónica en Chile al 31 de agosto de 2009. Dr Hugo Poblete B., Sociedad Chilena de Nefrología. 2009. *PMP: pacientes hemodializados por millón de población.

y $15,2 \%$ en los mayores de 64 años de edad) y una prevalencia de $34 \%$ de hipertensión arterial en la población general ${ }^{5}$. Por su parte, la evaluación de prevalencia de enfermedad renal en esta encuesta, reveló que $6,7 \%$ de los encuestados presentaba una creatininemia anormalmente elevada y en $14 \%$ era detectable la presencia de proteinuria.

Enfrentados a la alta incidencia de daño renal en la diabetes, los países desarrollados han diseñado estrategias para identificar los factores de riesgo que pueden conducir a que estos pacientes desarrollen nefropatía progresiva, lográndose identificar como los más importantes el mal control glicémico y la hipertensión arterial. El curso natural de la enfermedad renal en la diabetes es bastante conocido y mucho más identificable en la forma insulinodependiente por cuanto su inicio es más fácil de identificar. Lamentablemente la enfermedad renal asociada a la diabetes mellitus tipo 2 , su forma más frecuente, se desarrolla bastantes años después de instalado el defecto del control de la glicemia y en una forma absolutamente asintomática, lo que dificulta hacer un diagnóstico oportuno. Si bien inicialmente existe una función renal normal o incluso aumentada por efecto de una hiperfiltración mediada hemodinámicamente, a medida que progresa el daño renal se va perdiendo la permeabilidad selectiva de la barrera de filtración glomerular y se empieza a detectar la presencia anormal de pequeñas cantidades de proteína en la orina, inicialmente como albúmina (fase microalbuminúrica) para luego incrementar en cantidad (macroalbuminuria) y finalmente diversificar su calidad y aumentar en cantidad (proteinuria nefrótica). Si no son intervenidas precozmente, estas alteraciones derivan irreversiblemente en la pérdida progresiva de función renal general, apareciendo a la larga los síntomas y signos de insuficiencia renal crónica que requieren terapias sustitutivas como la diálisis o el trasplante renal para prolongar la vida del paciente. En promedio, la enfermedad renal en la diabetes tipo 2 se hace evidente después de 15-20 años de establecida la diabetes, siendo infrecuente antes de los 10 años y, curiosamente, aquellos pacientes que no la evidencian después de 25 años es raro que la presenten posteriormente.

Con estas consideraciones, resulta evidente que será necesario tener una vigilancia adecuada para el diagnóstico precoz de daño renal en el paciente diabético y para ello resultan fundamentales dos recursos: la detección precoz de albúmina en la orina y la estimación de la velocidad de filtración glomerular a través de la medición de la creatininemia. Estos dos recursos diagnósticos han sido simplificados, pudiéndose medir adecuadamente la albuminuria en una muestra aislada matinal corrigiéndola por la creatininuria; con respecto a la velocidad de filtración glomerular, existen fórmulas validadas 
que permiten estimarla adecuadamente a partir de la creatininemia, sexo, peso y otras variables no invasivas obtenidas del paciente. La Asociación Americana de la Diabetes y el Instituto de Salud Pública de los Estados Unidos de Norteamérica recomiendan la determinación de estos dos marcadores clínicos al menos una vez al año en todo paciente reconocido como diabético.

Teniendo en cuenta la importancia de la hipertensión, resulta interesante destacar que se ha logrado demostrar que es posible intervenir en la evolución natural de la nefropatía diabética retardando su progresión mediante el uso de fármacos antihipertensivos que antagonizan el sistema renina-angiotensina ${ }^{6}$, como los inhibidores de la enzima convertidora de angiotensina y los bloqueadores del receptor de angiotensina. Estos fármacos, que tienen una ventaja de costoeficiencia ${ }^{7}$, son capaces, además, de disminuir la proteinuria (un marcador clínico de progresión por excelencia) independiente de su efecto antihipertensivo, reducir el riesgo cardiovascular general y podrían incluso reducir la incidencia de nefropatía diabética ${ }^{8}$. En la población con diabetes tipo 2, la inhibición del sistema reninaangiotensina-aldosterona con estos fármacos ha logrado disminuir la progresión de normoalbuminuria a microalbuminuria (prevención primaria), disminuir la progresión de microalbuminuria a macroalbuminuria (prevención secundaria) y retardar el desarrollo de enfermedad renal terminal (prevención terciaria). Sin perjuicio de lo anterior, se ha podido establecer que el uso de otros tipos de antihipertensivos, por el sólo hecho de controlar las cifras tensionales tienen también un rol benéfico en la progresión de esta enfermedad.

El manejo dietético es sin duda un elemento terapéutico fundamental para reducir los efectos de la diabetes sobre la función renal, especialmente en etapas precoces. Un tratamiento normoglicemiante intensivo con objetivos exigentes de hemoglobina glicosilada, es capaz de reducir la frecuencia de aparición y detener la progresión de la nefropatía diabética una vez evidenciada9 ${ }^{9}$. Un mayor énfasis en este aspecto se ha observado con los hallazgos de regresión de la nefropatía diabética mediante un excepcional control metabólico, como el que se puede lograr por un trasplante exclusivo del páncreas $^{10}$. Estas medidas pueden tener aún mayor éxito si se complementan con una reducción en el excesivo consumo de proteínas, llevándolo a niveles moderados, y un adecuado manejo de la hiperlipidemia, trastorno asociado de alta frecuencia que colaborará también en la reducción del alto riesgo cardiovascular de estos pacientes.

Con todo el esfuerzo anteriormente descrito, existe un número no despreciable de pacientes que inevitablemente requerirán terapias de sustitución. En esa circunstancia debe tenerse en cuenta que ni la hemodiálisis ni el trasplante renal aislado solucionan todos los problemas del paciente diabético con nefropatía, y que éste se mantiene en una condición de alto riesgo cardiovascular.

El conocimiento de los altos gastos derivados de las terapias de sustitución de la función renal, comparado con la efectividad y bajo costo de las medidas orientadas a la detección precoz del daño renal en la diabetes y su oportuna intervención dietética y farmacológica obligan a la comunidad médica a una actitud preventiva que evite la obesidad y el sedentarismo tan asociados a ella, a una actitud de pesquisa precoz de la diabetes misma y su temida complicación renal de tal modo de contener los costos personales, sociales y económicos asociados a esta enfermedad y sus complicaciones.

La efectividad de estas medidas pasa por la realización de campañas de prevención del desarrollo de la diabetes tipo 2, una educación médica que incluya en los planes de estudio la detección precoz de la enfermedad renal diabética a través de la microalbuminuria y la medición de la velocidad de filtración glomerular, una educación masiva de la población respecto del significado de la diabetes, de su adecuado control y del conocimiento de sus complicaciones y, finalmente, el acceso oportuno al manejo terapeútico eficaz destinado a prevenir y/o retardar la evolución de la enfermedad.

Aprovechemos el día mundial del riñón para iniciar este esfuerzo nacional conjunto cuyos resultados nos agradecerán las generaciones futuras.

\section{Referencias}

1. Zimmet P, Alberti KG, Shaw J. Global and societal implications of the diabetes epidemic. Nature 2001; 414: 782-7.

2. King H, Aubert RE, Herman WH. Global burden of diabetes, 1995-2025: prevalence, numerical estimates, and projections. Diabetes Care 1998; 21: 1414-31. 
3. Bakris GL, Ritz E. The message for World Kidney Day 2009: hypertension and kidney disease: a marriage that should be prevented. Kidney Int 2009; 75: 449-52.

4. United States Renal Data System. USRDS 2007. Annual Data Report. Bethesda, MD: National Institute of Diabetes and Digestive and Kidney Diseases, National Institutes of Health, U.S. Department of Health and Human Services; 2007.

5. Escobar C, Arce I, Jara A, Mezzano S, Ardiles L. Renal health in Chile. Ren Fail 2006; 28: 639-41.

6. Ruggenenti P, Fassi A, Ilieva AP, Bruno S, Iliev IP, Brusegan V, et al. Preventing microalbuminuria in type 2 diabetes. N Engl J Med 2004; 351: 1941-51.

7. Ravera M, Re M, Vettoretti S. Economic evaluation of angiotensin receptor blockers in type 2 diabetes, hypertension, and nephropathy. J Am Soc Nephrol 2006; 17: S44-S48.

8. Strippoli GF, Craig MC, Schena FP, Craig JC. Role of blood pressure targets and specific antihypertensive agents used to prevent diabetic nephropathy and delay its progression. J Am Soc Nephrol 2006; 17: S153-S155.

9. Patel A, Macmahon S, Chalmers J, Neal B, Billot L, Woodward $\mathrm{M}$, et al. Intensive blood glucose control and vascular outcomes in patients with type 2 diabetes. $\mathrm{N}$ Engl J Med 2008; 358: 2560-72.

10. Fioretto P, Steffes MW, Sutherland DE, Goetz FC, Mauer M. Reversal of lesions of diabetic nephropathy after pancreas transplantation. N Engl J Med 1998; 339: 69-75. 